\title{
Stability windows and unstable root-loci for linear fractional time-delay systems
}

\author{
André R. Fioravanti* Catherine Bonnet* Hitay Özbay ** \\ Silviu-Iulian Niculescu ${ }^{* * *}$ \\ * INRIA Saclay - Ilê-de-France, Supélec, 3 rue Joliot Curie, 91192, \\ Gif-sur-Yvette, France. (e-mail: \{andre.fioravanti, \\ catherine.bonnet\}@inria.fr). \\ ** Bilkent University, Department of Electrical and Electronics \\ Engineering, Ankara 06800,Turkey.(e-mail: hitay@bilkent.edu.tr) \\ *** L2S (UMR CNRS 8506), CNRS-Supélec, 3 rue Joliot Curie, 91192, \\ Gif-sur-Yvette, France. (e-mail: Silviu.Niculescu@lss.supelec.fr)
}

\begin{abstract}
The main point of this paper is on the formulation of a numerical algorithm to find the location of all unstable poles, and therefore the characterization of the stability as a function of the delay, for a class of linear fractional-order neutral systems with multiple commensurate delays. We start by the asymptotic position of the chains of poles and conditions for their stability, for a small delay. When these conditions are met, we continue by means of the root continuity argument, and using a simple substitution, we can find all the locations where roots cross the imaginary axis. We can extend the method to provide the location of all unstable poles as a function of the delay. Before concluding, some examples are presented.
\end{abstract}

Keywords: Delay Effects, Fractional Systems, Neutral Systems, Root-Locus.

\section{INTRODUCTION}

Time-delay systems have a major importance both in the theoretical and practical domains, and this can be seen by the large amount of articles dealing with many different problems for this class of systems. Since the initial studies of [Pontryagin [1955]] and [Bellman and Cooke [1963]] many results have been achieved, specially over the last decades, as it can be seen, among many others, in the book [Niculescu and $\mathrm{Gu}$ [2004]] and the survey paper [Richard [2003]], and references therein. Similarly, fractional order systems are also obtaining large attention in the literature in the last years since they offer an excellent fit to the data in many situations as, for example, in biophysics, thermodynamics and rheology, see [Hilfer [2000]] and references therein.

One of the basic questions about any dynamical systems is stability. With respect to systems with delay, we can go further, and be interested on how this property will vary if we increase the value of the delay. It is known that an interesting phenomenon, namely stability windows, might happen. There has been a large effort to deal with this problem, as can be seen by the large quantity of articles dealing with it for the standard case; see [Walton and Marshall [1987]], [Olgac and Sipahi [2004]], and many others. Recently, [Fioravanti et al. [2010a]] brought another numerical technique to deal with this problem, and further extended its capabilities by using the results obtained to find the position of the unstable poles. In fact, in the present work, we expand the results obtained in [Fioravanti et al. [2010a]] for the class of fractional systems with delay.
Although the method of [Walton and Marshall [1987]] can be successfully expanded to cope with multiple delays and also fractional systems, each extra commensurate term of the delay after the first one needs to be reduced and this process potentially doubles at each step the degree of the polynomial we need to solve. So, even if we are dealing with low degree system with multiple delays, this method will require the zeros of a high order polynomial, which can be a challenging and perhaps unreliable numerical problem.

Recently, there has been some development of new methods dealing with fractional order system with delays. In [Hwang and Cheng [2006]], a numerical procedure based on Cauchy's integral theorem was proposed to test the stability of such systems, and in [Hwang and Cheng [2005]], a technique based on the Lambert $W$ function was used for the same purpose.

For neutral systems, one needs to take extra care due to the small-delay effect. As it can be seen in [Hale and Lunel [2001]], this splits the systems between two classes. One that has the same number of unstable poles when the delay passes from 0 to $0^{+}$, and for that has a chance of being stable for a non-zero value of the delay, and the other where infinitely many unstable poles appear when the delay varies from 0 to $0^{+}$. Even if the delay free system is stable, these systems cannot recover stability for any finite positive delay. This phenomenon is also present for fractional delay systems [Fioravanti et al. [2010b]].

The rest of the paper is organized as follows. Section 2 contains the problem statement and assumptions. Section 3 brings some stability tests for the type of systems we are dealing with, and in Section 4 , based on a pseudo- 
delay transformation, we develop a numerical procedure that identifies the stability zones as a function of the delay. The location of the unstable poles as well as their variation as a function of the delay is also given. Section 5 brings some examples to illustrate the results presented and finally Section 6 concludes the work.

The notation used throughout is standard. The set of natural numbers is denoted by $\mathbb{N}$, whereas $\mathbb{N}_{N}$ denotes the set of its first $N$ elements (i.e., $\mathbb{N}_{N}=\{1, \ldots, N\}$ ). $\mathbb{C}\left(\mathbb{C}^{+}, \mathbb{C}^{-}\right)$is the set of complex numbers (with strictly positive, and strictly negative real parts), and $\jmath=\sqrt{-1}$ is the imaginary unit. For $z \in \mathbb{C}, \bar{z}$ denotes its complex conjugate, and $\angle z, \Re(z)$ and $\Im(z)$ define the argument (taken here from $(-\pi, \pi])$, the real part and the imaginary part of $z . \mathbb{R}\left(\mathbb{R}^{+}, \mathbb{R}^{-}\right)$denotes the set of real numbers (larger or equal to zero, smaller or equal to zero).

\section{PROBLEM FORMULATION}

Consider a system that in the frequency-domain has the following characteristic equation

$$
C(s, \tau)=p\left(s^{\alpha}\right)+\sum_{k=1}^{N} q_{k}\left(s^{\alpha}\right) e^{-k s \tau},
$$

where the parameter $\tau$ is non-negative, $p\left(s^{\alpha}\right)$ and $q_{k}\left(s^{\alpha}\right)$ for $k \in \mathbb{N}_{N}$ are polynomials in $s^{\alpha}$ with $\alpha \in(0,1)$ and $\operatorname{deg} p \geq \operatorname{deg} q_{k}$. Here, the degree is interpreted as the degree in $s^{\alpha}$, and therefore it is an integer. If $\operatorname{deg} p=\operatorname{deg} q_{k}$ for at least one $k \in \mathbb{N}_{N}$, then equation (1) defines a neutral time-delay system, otherwise it will consist of the retarded type. As it will be seen in sequence, the analysis of the former is more involved and will require one extra step. Notice that the zeros of the characteristic equation (1) are the poles of the system under consideration. Therefore, in the sequel, we will use both terms indistinctly. Furthermore, we assume that:

Assumption 1. The polynomials $p(s), q_{1}(s), \ldots, q_{N}(s)$ do not have any common zero.

It is obvious that if Assumption 1 is violated, then a common factor $c(s) \neq$ constant can be factored out of the characteristic polynomial (1). These poles will be present for all the values of $\tau$, and therefore, if any of the poles of $c(s)$ is unstable, the system will be unstable for all values of the delay, whereas if all the poles are stable, they do not play a significant role in the stability test. In this case, simplifying by $c(s)$ we get a system described by (1) satisfying Assumption 1.

Assumption 2. The polynomials $p(s)$ and $q_{k}(s)$ satisfy

$$
p(0)+\sum_{k=1}^{N} q_{k}(0) \neq 0 \text {. }
$$

This means that $s=0$ is not a root of (1) for all values of $\tau$. At the same time, this assumption also guarantees that roots at $s=0$ can only happen for $\tau \rightarrow \infty$. This property will be very important in the following sections.

\section{STABILITY OF FRACTIONAL-ORDER SYSTEMS WITH DELAY}

We work here in an input-output framework and consider $H_{\infty}$-stability, that is, the system has a finite $L_{2}(0, \infty)$ gain if

$$
\|G\|_{H_{\infty}}=\sup _{u \in L^{2}, u \neq 0} \frac{\|G u\|_{L^{2}}}{\|u\|_{L^{2}}}<\infty,
$$

and we recall that $H_{\infty}\left(\mathbb{C}_{+}\right)$is the space of functions which are analytic and bounded in the open right half-plane $\mathbb{C}_{+}$.

The BIBO-stability (finite $L_{\infty}$-gain) of fractional systems with delays has been considered in [Bonnet and Partington [2002]] where it is shown that BIBO stability conditions already known for delay systems can be extended to the case of fractional delay systems. As BIBO-stability implies $H_{\infty}$-stability (see [Mäkilä and Partington [1993], Partington and Mäkilä [1994]]), similar results can be derived immediately for $H_{\infty}$-stability.

From [Bonnet and Partington [2002], Theorem 3.2] we get that systems with transfer function with denominator of the form (1) and of retarded type are $H_{\infty}$-stable if and only if they do not have any poles in $\Re(s) \geq 0$ (in particular, no poles of fractional order at $s=0$ ). Proposition 3.3 of the same work gives a sufficient condition of $H_{\infty}$-stability for systems with transfer function of the form (1) and of neutral type: if there exists $a<0$ such that $P$ has no poles in $\left(\mathbb{C} \backslash \mathbb{R}_{-}\right) \cap\{\Re(s)>a\} \cup\{0\}$ then $P$ is BIBO-stable. $H_{\infty^{-}}$ stability conditions for systems with poles asymptotically approaching the imaginary axis are still under study, as, for instance, [Fioravanti et al. [2010b], Partington and Bonnet [2004]], among others.

For fractional-order systems, a practical test for stability can be achieved if we use the variable substitution $\varsigma=s^{\alpha}$. Applying this substitution, the characteristic equation (1) becomes

$$
C_{\varsigma}(\varsigma, \tau)=p(\varsigma)+\sum_{k=1}^{N} q_{k}(\varsigma) e^{-k \varsigma^{1 / \alpha} \tau},
$$

This will transform the domain of the system from a multisheeted Riemann surface into the complex plane, where the poles can be easier calculated. In this new variable, the instability region of the original system is not given by the right half-plane, but in fact by the region described as:

$$
|\angle \varsigma| \leq \alpha \frac{\pi}{2}
$$

with $\varsigma \in \mathbb{C}$, as illustrated by Figure 1 .

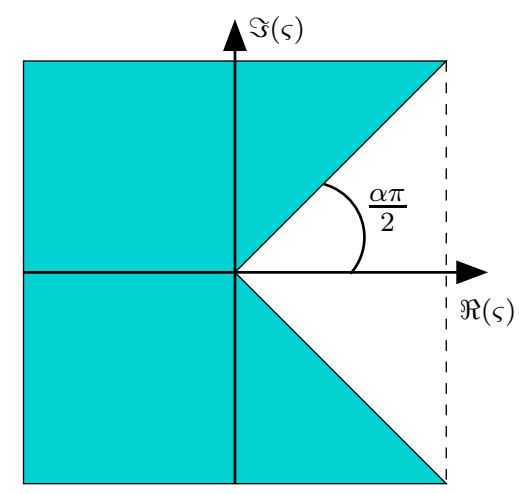

Figure 1. The $\varsigma$-stability region for fractional systems

Notice that under this transformation, the imaginary axis in the $s$-domain is mapped into the lines

$$
\angle \varsigma= \pm \alpha \frac{\pi}{2},
$$


in the $\varsigma$-domain, and therefore a solution $\varsigma^{\star}=\left|\varsigma^{\star}\right| \angle \pm \alpha \pi / 2$ implies that the original system has a purely imaginary solution of the type

$$
s^{\star}= \pm j\left|\varsigma^{\star}\right|^{\alpha^{-1}} .
$$

Also, we can notice that every solution $\varsigma^{*}$ such that $\left|\angle \varsigma^{*}\right|<\alpha \pi$ is mapped into the physical Riemann sheet in the $s$-domain through the inverse transform $s=\varsigma^{1 / \alpha}$.

\section{NUMERICAL PROCEDURE}

The main objective of this section is to define a procedure able to calculate the values of $\tau$ where there exists a crossing of poles through the imaginary axis. With these values in hand, we will be able to calculate the direction of crossing from the left half plane to the right one, which we will denote as a destabilizing crossing, or from the right to the left, meaning this is a stabilizing crossing. Notice that the use of the expressions destabilizing and stabilizing crossings means only that a pair of poles is crossing the imaginary axis in the defined direction, and not that the system is turning unstable or stable, respectively. For that, it is necessary to know the number of unstable poles before the crossings.

Compared to the work presented in [Fioravanti et al. [2010a]], the one proposed here is much more involved. One major difference is the fact that the roots of the characteristic equation can cross from one Riemann sheet to the other, which makes the procedure of following the roots in the $s$-domain much more complicated. To circumvent this complexity, we first apply the transformation to work in the s-domain, and after completing the root-locus we map just part of the result which will fall back into the physical Riemann sheet of the $s$-domain. But as it will be clear in the sequel, this transformation changes the form of the delay term, and this must be taken into account.

\subsection{Stability for $\tau=0$}

In the same way as many other procedures, we need to start by the study of the delay free system. Considering $\tau=0$ in (4), we get a polynomial with real coefficients, whose roots can be easily found. We will denote the number of unstable poles of $C_{\varsigma}(\varsigma, 0)$ as $N_{u}$ and their location $\varsigma_{k}$ for $k \in \mathbb{N}_{N_{u}}$, since this information will be crucial in the following developments.

\subsection{Location of Chains of Poles}

If the system we are dealing with is of the retarded type, all the infinite new poles that appear when we pass from $\tau=0$ to $\tau=0^{+}$will be in the extreme left half-plane, that means, $\Re(s) \rightarrow-\infty$, and so this step can be ignored. The case of neutral systems needs to be dealt with care. We will follow the steps from [Fioravanti et al. [2010b]] for the characterization of the asymptotic behavior of the chains of poles:

Proposition 1. Let $C(s, \tau)$ be the characteristic equation of a neutral system defined as in (1). Consider the first order approximation

$$
\frac{q_{k}(s)}{p(s)}=a_{k}+\mathcal{O}\left(s^{-\alpha}\right) \quad \text { as } \quad|s| \rightarrow \infty,
$$

where, denoting $\operatorname{deg} p=d, p(s)=\sum_{m=0}^{d} \rho_{m} s^{m}$ and $q_{k}(s)=\sum_{m=0}^{d} \sigma_{k, m} s^{m}$, equation (8) is satisfied with

$$
a_{k}=\frac{\sigma_{k, d}}{\rho_{d}} .
$$

Now let $M \leq N$ be the greatest integer such that $a_{M} \neq 0$. The neutral chains of poles asymptotically approach the vertical lines

$$
\Re(s)=-\frac{\ln (|r|)}{\tau}
$$

for each solution $r$ of the polynomial equation

$$
1+\sum_{k=1}^{M} a_{k} z^{k}=0
$$

Proof: See [Fioravanti et al. [2010b]] and references therein.

Equations (10) and (11) provide an easy-to-check condition in order for the neutral chains of poles be in the left half-plane. For this, all roots $r$ of (11) must have $|r|>1$.

In case any $|r|<1$, then at least one chain of poles will always be in the right half-plane. That means that the system can not be stable for any positive value of $\tau$. The intermediate case, where we have all $|r| \geq 1$, with the equality holding for at least one root of (10), needs to be further explored to determine from which side of the imaginary axis the poles are. But it is important to recall that in this case, the rule "no poles in the close right halfplane" is no longer sufficient to guarantee $H_{\infty}$-stability, and other properties must be checked. Indeed, the characteristic equation does not provide the full information necessary to state stability in this case, and we need to work with the complete transfer function. We invite the reader to see [Fioravanti et al. [2010b]] for further insights on this subject.

Assumption 3. From now on, we will consider that there are no chains of poles in the extended right half-plane (i.e., there exists a finite number of poles in $\Re(s)>-a$ for some $a>0)$.

\subsection{Crossing Position}

In order to find the location in the imaginary axis where the crossings occur, we will rely on a transformation of variables which decouples the polynomials and the exponential part. Since for $s=\jmath \omega$ the exponential terms in (1) will have modulus equal to one, the idea is to replace $e^{-\jmath \omega \tau k}$ with $e^{-\jmath \theta k}$ and find the roots of the resulting complex pseudo-polynomial as a function of $\theta \in[0, \pi]$. In other words, we find all the roots of the complex pseudopolynomial in $s$

$$
\widetilde{C}(s, \theta)=p\left(s^{\alpha}\right)+\sum_{k=1}^{N} q_{k}\left(s^{\alpha}\right) e^{-j k \theta},
$$

varying $\theta$ in the interval $[0, \pi]$.

Lemma 1. For any $\tau$ and $s=\jmath \omega$ such that $\widetilde{C}(s, \theta)=0$, then $\widetilde{C}(\bar{s}, 2 \pi-\theta)=0$.

Proof: It is a matter of simple substitution to show that $\widetilde{C}(\jmath \omega, \theta)=\overline{\widetilde{C}(-\jmath \omega, 2 \pi-\theta)}$. 
Lemma 2. There exist $s=\jmath \omega$ and $\tau>0$ such that $C(s, \tau)=0$ if and only if there exists $\theta \in[0, \pi]$ such that $\widetilde{C}(s, \theta)=0$.

Proof: For the sufficiency, recall that by the Assumption 2 the case $\omega=0$ can be neglected. So letting

$$
\tau(\omega, \theta, \ell)=\frac{\theta}{\omega}+\frac{2 \pi \ell}{\omega}
$$

and choosing $\ell=\{0,1, \ldots\}$ if $\omega>0$ or $\ell=\{-1,-2, \ldots\}$ if $\omega<0$ will provide $\tau>0$ such that (1) is satisfied.

For the necessity, choose $\theta$ as $\angle e^{\jmath \omega \tau}$, taken from $[0, \pi]$, and notice that

$$
e^{-\jmath k \omega \tau}=e^{-\jmath k \theta}
$$

for all $k \in \mathbb{N}$.

It is important to notice that for a fixed $\theta \in[0, \pi], \widetilde{C}$ is a pseudo-polynomial with complex coefficients, but no delays. But as it was stated before, with the variable transformation $\varsigma=s^{\alpha}$, there is a direct relation between the roots on the imaginary axis for the $s$-domain with the ones having argument $\pm \alpha \pi / 2$ in the $\varsigma$-domain. We must also recall that, due to the variable transformation in the exponential term, a solution $s^{\star}$ of $\widetilde{C}$ does not imply that $\overline{s^{\star}}$ is another solution. On the other hand, since by the Assumption 2 we can neglect the roots at the origin, all roots in the imaginary axis of (1) occur in complex conjugate pairs, and so we can finally state our main result.

Theorem 1. Let Assumptions 1-3 hold. Let $\Omega$ be the set of all ordered pairs $(\omega, \theta)$, with $\omega \in \mathbb{R}$ and $\theta \in[0, \pi]$ such that $\widetilde{C}(\jmath \omega, \theta)=0$ and

$$
\tau(\omega, \theta, \ell)=\frac{\theta}{\omega}+\frac{2 \pi \ell}{\omega}
$$

for all $(\omega, \theta) \in \Omega$. Choose $\ell=\{0,1, \ldots\}$ if $\omega>0$, and $\ell=\{-1,-2, \ldots\}$ if $\omega<0$. Let $\Delta$ be defined as the set of all the ordered pairs $( \pm \jmath \omega, \tau(\omega, \theta, \ell))$. Then $\Delta$ is the complete set of roots of (1) on the imaginary axis for $\tau>0$.

Proof: First we need to show that any element of $\Delta$ is a root for (1). The result for the term " $+\omega$ " comes directly from the sufficiency of Lemma 2, whereas the " $-\omega$ " comes from the aforementioned fact that the poles in the imaginary axis of (1) occur in complex conjugate pairs. Finally, it lacks to show that any root of (1) is an element of $\Delta$. But from Lemma 1 , we see that for the complex conjugated solutions of $\widetilde{C}(\jmath \omega, \theta)=0$, at least one of the $\theta$ will be in the $[0, \pi]$ interval. The rest follows from the necessity of Lemma 2 .

With these results in hand, it is easy to check if a system is stable independent of delay.

Corollary 1. If the system given by (1) is stable for $\tau=0$ and $\Omega=\emptyset$, then the system is stable for all positive values of $\tau$.

Proof: Direct from the fact that there are no roots crossing the imaginary axis.

The set $\Omega$ together with the root tendency of each ordered pair is what we call the root cluster. The root tendency will be better explained in the sequel, but as in the nonfractional case [Olgac and Sipahi [2004]], it is constant with respect to any sequential crossings in (15).
To be able to use the results of Theorem 1 , we need to be able to find all $\omega \in \mathbb{R}$ and $\theta \in[0, \pi]$ such that $\widetilde{C}(\jmath \omega, \theta)=0$. To this matter, we propose two distinct approaches.

The most direct one consists in sampling $\theta$ in its interval $[0, \pi]$, and for each fixed value $\theta^{\star}$, make the variable substitution $\varsigma=s^{\alpha}$, calculate the roots of the resulting complex polynomial in $\varsigma$

$$
\widetilde{C}_{\varsigma}\left(\varsigma, \theta^{\star}\right)=p(\varsigma)+\sum_{k=1}^{N} q_{k}(\varsigma) e^{-j k \theta^{\star}}
$$

Although we need to deal with a large number of polynomial equations to solve this problem, two points are relevant. First, all polynomials we need to solve will be of degree $n$ (for the system description given in (4)). Second, the root continuity argument still holds for $\widetilde{C}_{\varsigma}(\varsigma, \theta)$ as a function of $\theta$. As we have guaranteed that no poles in the origin will happen, this means that plotting the absolute value of the argument of those roots as a function of $\theta$ brings a useful graphic information. Also, as by assumption there are no chains of poles asymptotic to the imaginary axis, these plots will be continuous. Therefore, searching the positions where these curves cross the line $\theta=\alpha \pi / 2$ provide the information to calculate where the actual crossings through the imaginary axis are in the $s$ domain. This allows a better use of Newton's method in order to improve numerical accuracy without increasing the possibility of getting false results.

The second method we propose tries to keep the advantages of the previous one without increasing the computational burden. If $(\varsigma, \theta)$ is a simple root of $(16)$, then a small perturbation on $\theta^{\star}=\theta+\epsilon$ will provide a solution of $\widetilde{C}\left(\varsigma^{\star}, \theta^{\star}\right)=0$ with the form

$$
\varsigma^{\star}=\varsigma+\sum_{k=1}^{\infty} \lambda_{k} \epsilon^{k}
$$

where

$$
\lambda_{1}=\jmath \frac{\sum_{k=1}^{N} k q_{k}(\varsigma) e^{-\jmath \theta k}}{p^{\prime}(\varsigma)+\sum_{k=1}^{N} q_{k}^{\prime}(\varsigma) e^{-\jmath \theta k}}=T(\varsigma, \theta) .
$$

Here, $p^{\prime}(\varsigma)$ and $q_{k}^{\prime}(\varsigma)$ denote the derivative of the polynomials $p(\varsigma)$ and $q_{k}(\varsigma)$ in $\varsigma$ respectively.

So, a method to provide the same curves as the one calculated with the gridding method is to numerically integrate $T(\varsigma, \theta)$ for $\theta$ varying from 0 to $\pi$ and the starting positions of $\varsigma$ being the roots of $\widetilde{C}_{\varsigma}(\varsigma, 0)=0$, which are the same as the roots of $C_{\varsigma}(\varsigma, 0)=0$ already calculated for the test of stability in $\tau=0$. A possible strategy to integrate this function is to predict the next step by means of $T(\varsigma, \theta)$ to be close enough of the real solution and therefore being able to correct it with Newton's method. The size of the step can be tuned during execution by regarding the distance of the prediction part and the real solution of the correction one.

The cases where $T(\varsigma, \theta)=0$ or where we have multiple roots can still be dealt with, see [Chen et al. [2009a]] and [Chen et al. [2009b]]. But this will need a higher order analysis or a different approach for the definition of (17). On the other hand, in both cases, when this event happens, we can always stop the numerical integration, deal locally with the gridding method provided before, and re-start 
the integration method once we achieve simple roots and $T(\varsigma, \theta) \neq 0$.

\subsection{Direction of Crossing}

The objective now is to find for each crossing of roots through the imaginary axis, if it is a stabilizing or a destabilizing one. As it was shown in [Olgac and Sipahi [2002]], this is constant with respect to subsequential crossings ( $\ell$ in $(15)$ ), and therefore it is denoted as root tendency.

It is more convenient to calculate this point in the $s$ domain, but we can still deal with this problem in the same way we dealt before. Assume that $(s, \tau)$ is a simple root of $C(s, \tau)=0$. For a small variation of $\tau^{\star}=\tau+\epsilon$ then a solution of $C\left(s^{\star}, \tau^{\star}\right)=0$ can be found with the form

$$
s^{\star}=s+\sum_{k=1}^{\infty} \mu_{k} \epsilon^{k}
$$

where

$$
\begin{aligned}
\mu_{1} & =s \frac{\sum_{k=1}^{N} k q_{k}(s) e^{-\tau s k}}{\alpha s^{\alpha-1} p^{\prime}(s)+\sum_{k=1}^{N}\left(\alpha s^{\alpha-1} q_{k}^{\prime}(s)-\tau k q_{k}(s)\right) e^{-\tau s k}} \\
& =V(s, \tau) .
\end{aligned}
$$

The root tendency is given by $\operatorname{sign}(\Re(V(j \omega, \tau)))$, where $(j \omega, \tau) \in \Delta$. If it is positive, then it is a destabilizing crossing, whereas if it is negative, this means a stabilizing crossing. In case the result is 0 , a higher order analysis is needed, since this might be the case where the root just touches the imaginary axis and returns to its original halfplane.

To confirm the root tendency, we will show that

$$
\operatorname{sign}\left(\Re\left(V\left(j \omega, \frac{\theta}{\omega}+\frac{2 \pi \ell}{\omega}\right)\right)\right)
$$

is independent of $\ell$. Notice that for those given values, the exponential term

$$
e^{-\tau s k}=e^{-\jmath \theta k} e^{-\jmath 2 \pi \ell k}=e^{-\jmath \theta k}
$$

is independent of $\ell$. So, the only part $\ell$ still appears is in the denominator of $(20)$. But since $\operatorname{sign}(\Re(z))=$ $\operatorname{sign}(\Re(1 / z))$ for $z \in \mathbb{C}-\{0\}$, we can calculate the root tendency over the inverse of $V$, and we readily see that $\ell$ just enters in the imaginary part of it.

From this point, it is easy to determine for each value of the delay if the system has unstable roots or not. Start counting from the number of unstable poles of the delay free system. Sort $\Delta$ by the value of the delay of the crossing, and for each value of it such that the root tendency is positive, add one for the counting of unstable poles (they will always appear in pairs), or subtract one if the root tendency is negative. Repeat the procedure until the maximum of the delay. Finally, identify the values of $\tau$ where the number of unstable roots is zero. Those are the stable regions.

\subsection{Location of Unstable Poles}

Suppose now that the problem is not finding the values of $\tau$ such that the system is stable, but in fact finding the actual position of the unstable poles for a given value of delay $\tau^{\star}$. This can be achieved by an adaptation of the same techniques used before.

From the definition of $\Omega$, we can calculate a subset $\Delta_{\tau^{\star}}$ of $\Delta$ containing only the elements of $\Delta$ with the delay smaller than $\tau^{\star}$ and with positive root tendency.

To avoid any issues around the Riemann surfaces, it is more adequate to deal with this problem in the $\varsigma$-domain. So assuming that $(\varsigma, \tau)$ is a simple root of $C_{\varsigma}(\varsigma, \tau)=0$, then for a small variation of $\tau^{\star}=\tau+\epsilon$ then a solution of $C_{\varsigma}\left(\varsigma^{\star}, \tau^{\star}\right)=0$ can be found with the form $\varsigma^{\star}=\varsigma+$ $\sum_{k=1}^{\infty} \nu_{k} \epsilon^{k}$, where

$$
\begin{aligned}
\nu_{1} & =\frac{\varsigma^{1 / \alpha} \sum_{k=1}^{N} k q_{k}(\varsigma) e^{-\tau \varsigma^{1 / \alpha} k}}{p^{\prime}(\varsigma)+\sum_{k=1}^{N}\left(q_{k}^{\prime}(\varsigma)-\tau \alpha^{-1} \varsigma^{(1-\alpha) / \alpha} k q_{k}(\varsigma)\right) e^{-\tau \varsigma^{1 / \alpha} k}} \\
& =V_{\varsigma}(\varsigma, \tau) .
\end{aligned}
$$

Integrating $V_{\varsigma}(\varsigma, \tau)$ with respect to $\tau$ for each element of $\left(\left(s^{\circ}\right)^{\alpha}, \tau^{\circ}\right) \in \Delta_{\tau^{\star}}$ for $\tau \in\left[\tau^{\circ}, \tau^{\star}\right]$ and $\left(s^{\circ}\right)^{\alpha}$ as starting point, together with the unstable poles $\left(\varsigma_{k}\right)$ for the delay free system for $\tau \in\left[0, \tau^{\star}\right]$ will generate the curves of the root loci in the $\varsigma$-domain. Applying the inverse transformation $s=\varsigma^{1 / \alpha}$ for all the points in the $\varsigma$ root-loci with argument between $(-\alpha \pi, \alpha \pi)$ generates the root-loci in the physical layer in the $s$-domain. We can even back integrate from the elements of $\Delta_{\tau^{\star}}$ for $\tau \in\left[\tau^{\circ}, \tau^{\bullet}\right]$ in order to see their dynamics before the crossing, although back integrating until $\tau^{\bullet}=0$ can be a bad idea since there is a chance that this might lead to solutions asymptotic to $\Re(s) \rightarrow-\infty$. A good trade-off seems to be choosing $\tau^{\bullet}=\tau^{\circ} / 2$.

This procedure will provide the position of all unstable roots of (1), as well as some information about the stable ones. If one needs more information about the stable ones, one can always perform the same integration procedure over the stable roots for $\tau=0$, the stabilizing crossings, and even also back integrating from a number of future crossings. But no guarantee can be given that those will be the ones closer to the imaginary axis.

\section{EXAMPLES}

Our first example comes from [Hwang and Cheng [2006]]. Let us consider the system defined by

$$
C_{1}(s)=\frac{1}{(\sqrt{s})^{3}-1.5(\sqrt{s})^{2}+4 \sqrt{s}+8-1.5(\sqrt{s})^{2} e^{-\tau s}}
$$

Using a heavy computation scheme based on the Cauchy's integral, [Hwang and Cheng [2006]] showed that this system is unstable for $\tau=0.99$ but stable for $\tau=1$.

Applying the first part of the algorithm, we can see that there is a destabilizing cross of roots at $\tau=0.7854 k$ occurring at $s= \pm \jmath 8.0$ and a stabilizing cross at $\tau=$ $0.0499+0.9485 k$ for $s= \pm \jmath 6.6246$, for all $k \in\{0,1, \ldots\}$. Therefore, we have the following 5 stability windows; $0.0499<\tau<0.7854,0.9983<\tau<1.5708,1.9486<\tau<$ 2.3562, $2.8953<\tau<3.1416$ and $3.8437<\tau<3.9270$, which agrees with the result given by [Ozturk and Uraz [1985]] and in some sense explains it.

We further continue by considering $\tau=3.9$. As this value is inside the last stability window, we know beforehand that 
the system is stable. But we search a better understanding of the root-loci of the system as a function of the delay. Figure 2 brings this picture, where the colors represent the chosen $\tau$, with deep-blue for $\tau=0$ and strong red for $\tau=3.9$.

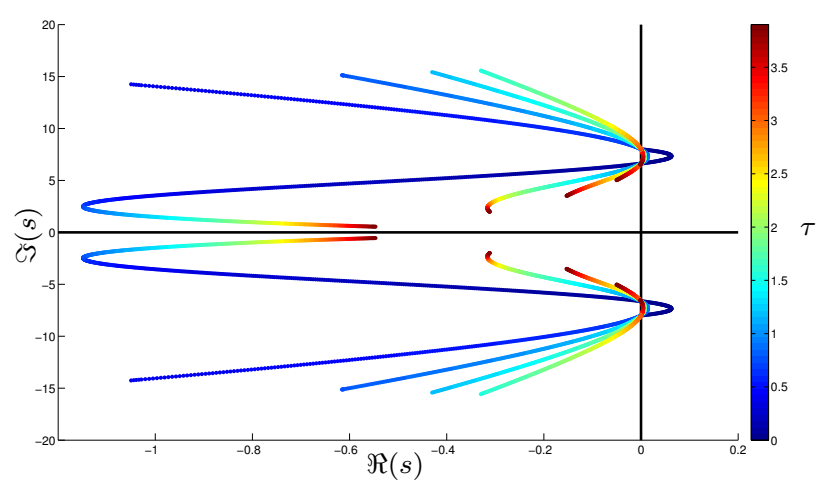

Figure 2. Root-loci for $C_{1}(s)$ until $\tau=3.9$

One can see that although the depth inside the right halfplane for each destabilizing cross becomes less expressive when $\tau$ increases, the stabilizing crossing happens closer to the following destabilizing one, and after the pair of poles cross at $\tau=3.9270$ to the right half-plane, the next pair of unstable poles arrive at $\tau=4.7124$ before the previous ones exit this half-plane at $\tau=4.7922$, and therefore the system cannot recover its stability.

The second example also comes from [Hwang and Cheng [2006]]. Considering the fractional-order system with two delays

$$
C_{2}(s)=\frac{1}{s^{5 / 6}+\left(s^{1 / 2}+s^{1 / 3}\right) e^{-0.5 s}+e^{-s}}
$$

It is stated that this system is stable. In order to apply our procedure, we transformed $C_{2}(s)$ into the following equivalent system

$$
\widetilde{C}_{2}(s, \tau)=\frac{1}{s^{5 / 6}+\left(s^{3 / 6}+s^{2 / 6}\right) e^{-\tau s}+e^{-2 \tau s}}
$$

and we have to study the stability for $\tau=0.5$.

This system has no unstable pole for $\tau=0$ (in fact, it has no pole in the physical Riemann sheet). Applying the methodology described before, we achieve that crossings through the imaginary axis happens for $\tau=2.3562+$ $6.2832 k$ and $\tau=2.6180+6.2832 k$, and both of them are destabilizing crosses. This means that the only stability window for this system is $0 \leq \tau<2.3562$. As $\tau=0.5$ is included in this window, we can ensure that the original system $C_{2}(s)$ is stable.

\section{CONCLUSION}

A new method for calculating stability windows and location of the unstable poles is proposed for a large class of fractional order time-delay systems. As the main advantages, we just deal with polynomials of the same order as that of the original system, and we have a graphical representation of the number of elements in the root cluster. Although there are still some implementation issues which need to be addressed, the results seem promising and consistent with the existing literature.

\section{REFERENCES}

R. Bellman and K. L. Cooke. Differential-difference equations. Academic Press, New York-London, 1963.

C. Bonnet and J. R. Partington. Analysis of fractional delay systems of retarded and neutral type. Automatica, 38:1133-1138, 2002.

J. Chen, P. Fu, S. I. Niculescu, and Z. Guan. When will zeros of time-delay systems cross imaginary axis? part 1: Eigenvalue series. Submitted, 2009a.

J. Chen, P. Fu, S. I. Niculescu, and Z. Guan. When will zeros of time-delay systems cross imaginary axis? part 2: Zero crossings. Submitted, 2009b.

A. R. Fioravanti, C. Bonnet, Özbay H, and S.-I. Niculescu. A numerical method to find stability windows and unstable poles for linear neutral time-delay systems. In Proceedings of the 9th IFAC Workshop on Time Delay Systems, Prague - Czech Republic, June 2010a.

A. R. Fioravanti, C. Bonnet, and H. Özbay. Stability of fractional neutral systems with multiple delays and poles asymptotic to the imaginary axis. In Proceedings of the 49th IEEE Conference on Decision and Control, Atlanta, USA, Dec. 2010b.

J. K. Hale and S. M. Verduyn Lunel. Effects of small delays on stability and control. Operator Theory; Advances and Applications, 122:275-301, 2001.

R. Hilfer, editor. Applications of Fractional Calculus in Physics. World Scientific, Singapore, 2000.

C. Hwang and Y. C. Cheng. A note on the use of the Lambert W function in the stability analysis of timedelay systems. Automatica, 41(11):1979-1985, 2005.

C. Hwang and Y. C. Cheng. A numerical algorithm for stability testing of fractional delay systems. Automatica, 42(5):825-831, 2006.

P. M. Mäkilä and J. R. Partington. Robust stabilizationBIBO stability, distance notions and robustness optimization. Automatica, 23(3):681-693, 1993.

S. I. Niculescu and K. Gu. Advances in Time-Delay Systems. Springer, 2004.

N. Olgac and R. Sipahi. An exact method for the stability analysis of time delayed LTI systems. IEEE Trans. Autom. Contro, 47(5):793-797, 2002.

N. Olgac and R. Sipahi. A practical method for analyzing the stability of neutral type LTI-time delayed systems. Automatica, 40:847-853, 2004.

N. Ozturk and A. Uraz. An analysis stability test for a certain class of distributed parameter systems with delays. IEEE Transactions on Circuits and Systems, 32(4):393 - 396, 1985.

J. R. Partington and C. Bonnet. $H_{\infty}$ and BIBO stabilization of delay systems of neutral type. Systems and Control Letters, 52:283-288, 2004.

J. R. Partington and P. M. Mäkilä. Worst-case analysis of identification-BIBO robustness for closed-loop data. IEEE Trans. Autom. Contro, 39:2171-2176, 1994.

L. S. Pontryagin. On the zeros of some elementary transcendental functions. Amer. Math. Soc. Transl, 2(1):95-110, 1955.

J. P. Richard. Time-delay systems: An overview of some recent advances and open problems. Automatica, 39: 1667-1694, 2003.

K. Walton and J. E. Marshall. Direct method for TDS stability analysis. IEE Proceedings, Part D, 134:101$107,1987$. 